\title{
Fractal dimensions of silica gels generated using reactive molecular dynamics simulations
}

\author{
Sudin Bhattacharya \\ Department of Mechanical Engineering, University of Michigan, Ann Arbor, Michigan 48109-2158 \\ John Kieffer ${ }^{\mathrm{a})}$ \\ Department of Materials Science and Engineering, University of Michigan, \\ Ann Arbor, Michigan 48109-2136
}

(Received 17 September 2004; accepted 15 December 2004; published online 1 March 2005)

\begin{abstract}
We have used molecular dynamics simulations based on a three-body potential with charge transfer to generate nanoporous silica aerogels. Care was taken to reproduce the sol-gel condensation reaction that forms the gel backbone as realistically as possible and to thereby produce credible gel structures. The self-similarity of aerogel structures was investigated by evaluating their fractal dimension from geometric correlations. For comparison, we have also generated porous silica glasses by rupturing dense silica and computed their fractal dimension. The fractal dimension of the porous silica structures was found to be process dependent. Finally, we have determined that the effect of supercritical drying on the fractal nature of condensed silica gels is not appreciable. () 2005 American Institute of Physics. [DOI: 10.1063/1.1857522]
\end{abstract}

\section{INTRODUCTION}

Over the last three decades, the sol-gel process has emerged as a particularly attractive way of manufacturing porous silica aerogels. The method makes it possible to control the geometry, porosity, and physical properties of the resulting structures by careful manipulation of the chemistry and processing parameters. ${ }^{1}$ The production of silica aerogels by the sol-gel method involves the hydrolysis and condensation of silicon alkoxide precursors of the form $\mathrm{Si}(\mathrm{O} R)_{4}(R$ is usually an alkyl group). The reaction consists of three stages: ${ }^{2}$ (1) hydrolysis, (2) alcohol condensation, and, (3) water condensation. The most commonly used precursors are tetramethoxysilane, $\mathrm{Si}\left(\mathrm{OCH}_{3}\right)_{4}$ (TMOS) and tetraethoxysilane, $\mathrm{Si}\left(\mathrm{OCH}_{2} \mathrm{CH}_{3}\right)_{4}$ (TEOS). In this paper we focus on stage (3) of the reaction- the water-producing condensation of fully hydrolyzed precursor molecules. This stage involves the polymerization of silicic acid $\left[\mathrm{Si}(\mathrm{OH})_{4}\right]$ monomers to produce the actual gel structure, along with water molecules as a byproduct.

The molecular dynamics (MD) simulation technique, which has been effectively used to study sol-gel reactions, ${ }^{3-6}$ offers an important advantage over experimental investigations, in that it affords a detailed insight into the atomic-scale phenomena that underlie the formation of a gel network. Unlike quantum mechanical calculations, which provide the most reliable description of atomic interactions, MD simulations based on classical potentials let us model configurations with sufficiently large number of atoms and for periods of time that accommodate elementary reaction processes.

Some investigators have followed an alternative route to the simulation of polymerization processes, i.e., through kinetic growth models of various sorts, ${ }^{7}$ including branching

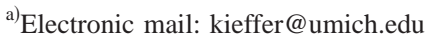

models, ${ }^{8}$ functional group models, ${ }^{9}$ bicomponent models, ${ }^{10}$ nearest-neighbor models, ${ }^{11}$ and cluster-cluster aggregation models. ${ }^{12-14}$ However these models involve a much higher level of abstraction, and unlike those based on MD, cannot explicitly account for the effects that result from changes in the chemistry of constituents.

The work presented here is based on the MD approach, using a reactive three-body interatomic potential that allows for charge transfer. Most potentials described in the literature are highly system specific, i.e., potential parameters may need to be adjusted when switching from one structural modification of a material to another. Our potential has been successfully applied to simulating the $\alpha$ and $\beta$ modifications of cristobalite, quartz, and amorphous silica, with one and the same set of parameters. This allowed us to investigate the $\alpha-\beta$ phase transition in cristobalite and quartz by direct simulation of the underlying process without preconception of the resulting structures, ${ }^{15}$ and to thereby uncover the nature of structural disorder in $\beta$ cristobalite. ${ }^{16} \mathrm{We}$ also discovered the structural transitions responsible for the thermomechanical anomalies of silica glass ${ }^{17}$ and for its permanent densification under high pressure. ${ }^{18}$ Here we show the applicability of this potential to the simulation of material systems in which structural changes are based on chemical reactions.

In addition to simulating the sol-gel reaction, we have examined the structural features of our simulated aerogels. The highly porous structure of these materials immediately suggests fractal (self-similar) scaling. Indeed, results from small-angle x-ray scattering ${ }^{19}$ (SAXS), neutron scattering ${ }^{20}$ (SANS), and nuclear magnetic resonance ${ }^{21}$ (NMR) studies support this hypothesis, and show that silica aerogels form fractal structures over more than two orders of magnitude in length. ${ }^{22}$ Unlike most computer simulations of fractal behavior in aerogels in the literature, which rely on reactionlimited, diffusion-limited, or ballistic cluster-cluster aggrega- 
TABLE I. Optimized potential parameters for $\mathrm{Si}, \mathrm{O}, \mathrm{H}$ interactions.

\begin{tabular}{|c|c|c|c|c|c|c|}
\hline Element & \multicolumn{2}{|c|}{$\sigma_{i}(\mathrm{~mm})$} & $n_{i}$ & \multicolumn{2}{|c|}{$z_{i}$} & $q_{i}^{0}(\mathrm{ecu})$ \\
\hline $\mathrm{Si}$ & \multicolumn{2}{|c|}{0.101} & 8 & \multicolumn{2}{|c|}{+4} & 0 \\
\hline $\mathrm{O}$ & \multicolumn{2}{|c|}{0.143} & 8 & \multicolumn{2}{|c|}{-2} & 0 \\
\hline $\mathrm{H}$ & \multicolumn{2}{|c|}{0.085} & 2 & \multicolumn{2}{|c|}{+1} & 0 \\
\hline Pair & $A_{i j}\left(10^{-19} \mathrm{~J}\right)$ & $\rho_{i j}\left(\mathrm{~nm}^{-1}\right)$ & $C_{i j}\left(10^{-19} \mathrm{~J} \mathrm{~nm}^{6}\right)$ & $\eta_{i j}\left(\mathrm{~nm}^{-1}\right)$ & $\kappa_{i j}\left(\mathrm{~nm}^{-1}\right)$ & $e^{\lambda^{i j}}$ \\
\hline $\mathrm{Si}-\mathrm{Si}$ & 0.1600 & 32.0000 & 0.00 & 0.00 & 32.0000 & 0.000 \\
\hline $\mathrm{Si}-\mathrm{O}$ & 0.1650 & 33.0000 & 0.00 & 3.20 & 33.0000 & 11.9499 \\
\hline $\mathrm{Si}-\mathrm{H}$ & 0.2150 & 31.8000 & 0.00 & 0.00 & 31.8000 & 0.000 \\
\hline $\mathrm{O}-\mathrm{O}$ & 0.2500 & 19.5000 & 0.00 & 0.00 & 19.5000 & 0.000 \\
\hline $\mathrm{O}-\mathrm{H}$ & 0.1000 & 34.5000 & $1.60 \mathrm{E}-06$ & 14.00 & 34.5000 & 124.1338 \\
\hline $\mathrm{H}-\mathrm{H}$ & 0.1900 & 34.5000 & 0.00 & 0.00 & 34.5000 & 0.00 \\
\hline \multicolumn{2}{|c|}{ Charge transfer } & $\delta_{i j}(\mathrm{ecu})$ & \multicolumn{2}{|c|}{$a(\mathrm{~nm})$} & \multicolumn{2}{|c|}{$b\left(\mathrm{~nm}^{-1}\right)$} \\
\hline \multicolumn{2}{|c|}{$\mathrm{Si}-\mathrm{O}$} & -0.25 & \multicolumn{2}{|c|}{0.27} & \multicolumn{2}{|c|}{80.00} \\
\hline \multicolumn{2}{|c|}{$\mathrm{O}-\mathrm{H}$} & 0.05 & \multicolumn{2}{|c|}{0.14} & \multicolumn{2}{|c|}{50.00} \\
\hline \multicolumn{2}{|c|}{ Triplet } & & \multicolumn{2}{|l|}{$\gamma_{i j k}\left(\operatorname{rad}^{-2}\right)$} & \multicolumn{2}{|c|}{$\bar{\theta}(\mathrm{rad})$} \\
\hline \multicolumn{2}{|c|}{$\mathrm{O}-\mathrm{Si}-\mathrm{O}$} & & \multicolumn{2}{|l|}{0.20} & \multicolumn{2}{|c|}{1.91} \\
\hline \multicolumn{2}{|c|}{ Si-O-Si } & & 0.45 & & \multicolumn{2}{|c|}{2.48} \\
\hline \multicolumn{2}{|c|}{$\mathrm{Si}-\mathrm{O}-\mathrm{H}$} & & 0.08 & & \multicolumn{2}{|c|}{2.48} \\
\hline \multicolumn{2}{|c|}{$\mathrm{H}-\mathrm{O}-\mathrm{H}$} & & 0.10 & & & \\
\hline
\end{tabular}

tion models, ${ }^{23-25}$ we have studied the self-similarity of aerogels generated by direct simulation of the chemical reactions that form these structures.

\section{POTENTIAL MODEL AND COMPUTATIONS}

Bonding in silica $\left(\mathrm{SiO}_{2}\right)$ exhibits significant ionic character. Pauling's electronegativity rule predicts the degree of ionicity in the bond to be about $45 \%$. This implies an extent of charge localization and directional character in the bond. It has been suggested that two-body central potentials are not suitable for the simulation of systems with directional bonding. ${ }^{26-28}$ Accordingly, we use a charge-transfer threebody potential incorporating a Coulomb term, a BornHuggins-Mayer repulsive term, ${ }^{29}$ and a three-body term that accounts for covalent interaction. For a single particle the potential energy is given by

$$
\begin{aligned}
\phi_{i}= & q_{i} \sum_{j=1}^{N} \frac{q_{j}}{4 \pi \varepsilon_{0} r_{i j}}+\sum_{j=1}^{N C} A_{i j}\left(1+\frac{z_{i}}{n_{i}}+\frac{z_{j}}{n_{j}}\right) e^{\left(\sigma_{i}+\sigma_{j}-r_{i j}\right) \rho_{i j}} \\
& +\sum_{j=1}^{N C-1} \sum_{k=j+1}^{N C}\left(\varphi_{i j}+\varphi_{i k}\right) e^{-\gamma_{i j k}\left(\bar{\theta}-\theta_{i j k}\right)^{2}}-\frac{C_{i j}}{r_{i j}^{6}} .
\end{aligned}
$$

In the first (Coulomb) term, $q_{i}$ denotes the charge on the $i$ th particle, $\varepsilon_{0}$ is the permittivity of free space, and $r_{i j}$, the distance between the $i$ th and $j$ th particles. The charge $q_{i}$ is evaluated as $q_{i}=q_{i}^{0}-2 \sum_{j=1}^{N C} \delta_{i j} \zeta_{i j}$ where $q_{i}^{0}$ is the charge of the isolated atom, $N C$ is the coordination number of the atom when bonded, $\delta_{i j}$ the amount of transferred charge, and $\zeta_{i j}$ $=\left(1+e^{\left(r_{i j}-a\right) b}\right)^{-1}$ is a function that modulates the transfer of charge with interatomic distance, $a$ and $b$ being empirical parameters. This provision of charge transfer allows for the formation and rupture of bonds in the reaction. Electroneu- trality is maintained by having $\delta_{j i}=-\delta_{i j}$. The long-range effects of the Coulomb interactions, resulting from their dependence on the inverse of $r_{i j}$, are evaluated using the Ewald summation approach. ${ }^{30,31}$

The remaining terms in Eq. (1) represent the BornHuggins-Mayer repulsive, the covalent attractive, and the van der Waals attractive components of the atomic interactions, in that order. The radial component of the covalent term is given by $\varphi_{i j}=-A_{i j}\left(1+z_{i} / n_{i}+z_{j} / n_{j}\right)$ $\times\left(\kappa_{i j} / \eta_{i j}\right) \zeta_{i j} e^{\lambda_{i j}} e^{-r_{i j} \eta_{i j}} . z_{i}$ and $n_{i}$ denote the valence and number of outer shell electrons of species $i$. The factors $A_{i j}, \sigma_{i}$, $\rho_{i j}, \kappa_{i j}, \eta_{i j}, \lambda_{i j}, C_{i j}$, and $\gamma_{i j k}$ are all empirical parameters; their numerical values are given in Table I. The chargetransfer function $\zeta_{i j}$ is of pivotal importance in this interaction potential. Not only does it control the redistribution of charge between species upon forming or breaking a covalent bond, it also modulates the strength of the covalent bond. Similarly, the angular restoring force described by $e^{-\gamma_{i j k}\left(\bar{\theta}-\theta_{i j k}\right)^{2}}$, where $\bar{\theta}$ is the equilibrium bond angle, enters the covalent bonding term conditionally, i.e., it is not active unless bonds between species $i, j$, and $k$ exist. Equation (1) was used to model all pair and three-body interactions involving $\mathrm{Si}, \mathrm{O}$, and $\mathrm{H}$ atoms, as indicated in Table $\mathrm{I}$.

We simulated condensation reactions using an ensemble of 512 silicic acid molecules (a total of 4608 particles) dispersed randomly in a cubic simulation box, with periodic boundary conditions applied. Some of the initial runs for validation purposes were carried out on a smaller system of 105 silicic acid molecules (945 particles). The molecules are relaxed at a temperature of $300 \mathrm{~K}$ to obtain a range of system densities by adjustment of the simulation box-varying from 1.0 to $2.0 \mathrm{~g} / \mathrm{cm}^{3}$ (vitreous silica has a density of $2.2 \mathrm{~g} / \mathrm{cm}^{3}$ ). These different configurations are then heated up to a tem- 

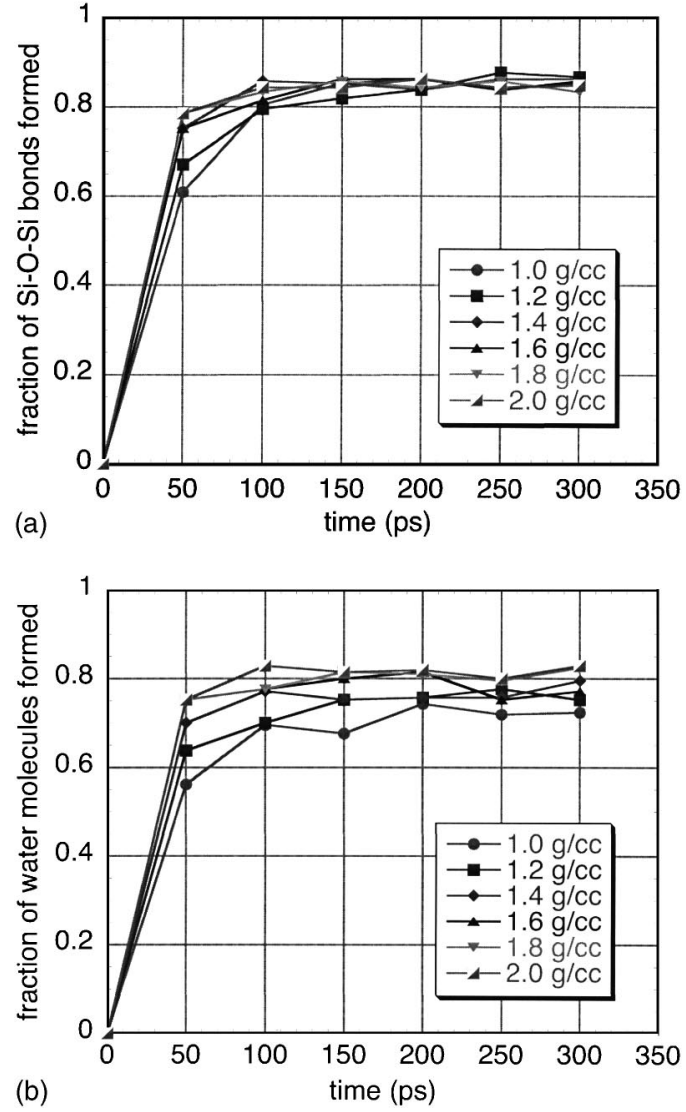

FIG. 1. (a) Degree of reaction for bridging siloxane ( $\mathrm{Si}-\mathrm{O}-\mathrm{Si})$ bonds; (b) degree of reaction for water formation (system temperature $=700 \mathrm{~K}$ ).

perature of $700 \mathrm{~K}$ to reproduce condensation reactions within a reasonable simulation time (reactions show a steady increase in rate from 300 to $700 \mathrm{~K}$ ). Configurations at each density are allowed to react for $300 \mathrm{ps}$ at $700 \mathrm{~K}$. The equations of motion are integrated using a time step $\Delta t$ $=0.001 \mathrm{ps}$. Water molecules formed as a result of the condensation reaction are initially allowed to stay in the system-thus the simulations resemble the actual production of aerogels in autoclaves under high temperature and pressure conditions with neutral $p \mathrm{H}$. The procedure followed is similar to that of Garofalini and Martin. ${ }^{3}$

\section{RESULTS AND DISCUSSION}

We begin by describing our observations on reaction rate behaviors, i.e., the time dependence of the degree of reaction, and its variation with temperature. The condensation reaction produces a polymerized silica network and water molecules. Figure 1 shows the degrees of formation of bridging siloxane ( $\mathrm{Si}-\mathrm{O}-\mathrm{Si}$ ) bonds and water. It is evident that siloxane bond formation at $700 \mathrm{~K}$ [Fig. 1(a)] is virtually independent of system density - all configurations in the range $1.0-2.0 \mathrm{~g} / \mathrm{cm}^{3}$ have attained $83-87 \%$ polymerization by $300 \mathrm{ps}$ (the degree of reaction is measured as the ratio of the number of siloxane bonds actually formed at a given time to the number stoichiometrically expected for a completed reaction). Water formation [Fig. 1(b)] shows a somewhat greater dependence on density and attains completion rates of $72-83 \%$ in the same time span-high-density configura-

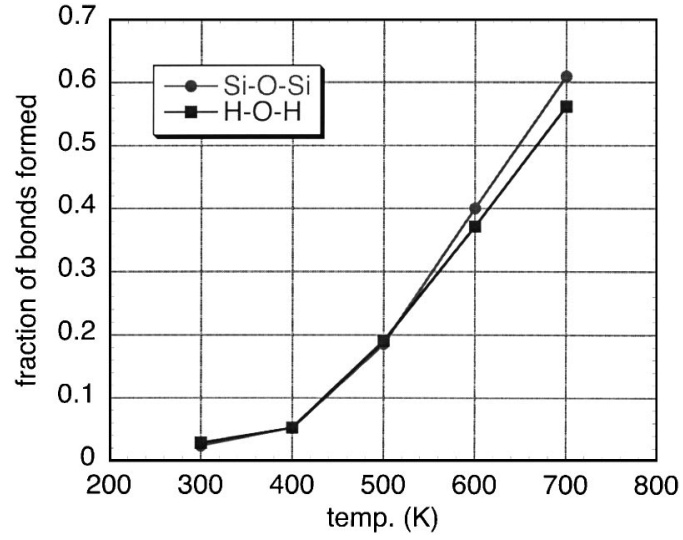

FIG. 2. Degree of polymer and water formation vs temperature (simulation time $=50 \mathrm{ps}$, system density $\left.=1.0 \mathrm{~g} / \mathrm{cm}^{3}\right)$.

tions being more reactive. Strictly speaking, it is not necessary to have the water molecules form to get a silica network structure-one merely needs hydrogen atoms and hydroxyl $(\mathrm{OH})$ groups to dissociate from the silicic acid monomers. It is therefore not surprising to observe different density dependences of the formation of polymer network and water molecules, even though the two processes are not independent of each other.

The temperature dependence of reaction rates was tested for the lowest-density configuration $\left(1.0 \mathrm{~g} / \mathrm{cm}^{3}\right)$. Figure 2 shows the fraction of polymer and water formed after $50 \mathrm{ps}$ - we see that the degree of formation for both $\mathrm{Si}-\mathrm{O}-\mathrm{Si}$ and $\mathrm{H}_{2} \mathrm{O}$ rises steeply with temperature. (The data in Fig. 2 reflects the increase in reaction kinetics, not the changes in equilibrium values of the degree of networking as a function of temperature.)

Both phenomenological models ${ }^{32}$ and MD simulation results $^{3-6}$ have indicated that the condensation reaction mechanism involves the dissociation of hydrogen ions from silicic acid monomers, followed by the formation of pentacoordinated silicon atoms and the rupture of a long $\mathrm{Si}-\mathrm{O}$ back bond to produce a dimer. We observe a similar process in our simulations. We have used a number of criteria to verify the realism of the simulated gel structures. Of particular interest is the structural evolution of the silica polymer network. We find in our simulations that the initial stages of condensation are marked by the formation of linear chainswhile closed rings are visible in the later stages of reaction. This observation is borne out by experimental results from NMR data and quantum calculations. ${ }^{33}$ The simulated networks exhibit a wide range of ring sizes, as one would expect for a fractal structure. Over the time scales covered by our simulations, the nature of the $\mathrm{Si}-\mathrm{O}$ network formed strongly depends on the density of the precursor solution. At low densities $\left(1.0-1.2 \mathrm{~g} / \mathrm{cm}^{3}\right)$ the condensed structures are characterized by separate clusters of molecules; while for denser configurations $\left(1.4-2.0 \mathrm{~g} / \mathrm{cm}^{3}\right)$, a single percolating cluster spanning the simulation box forms. We can also discern localized aggregates of atoms measuring on average between 10 and $20 \AA$, especially in the lowest-density configurations. However, these structural features exhibit a range of sizes, and at this point it is not clear how the constraint in the 


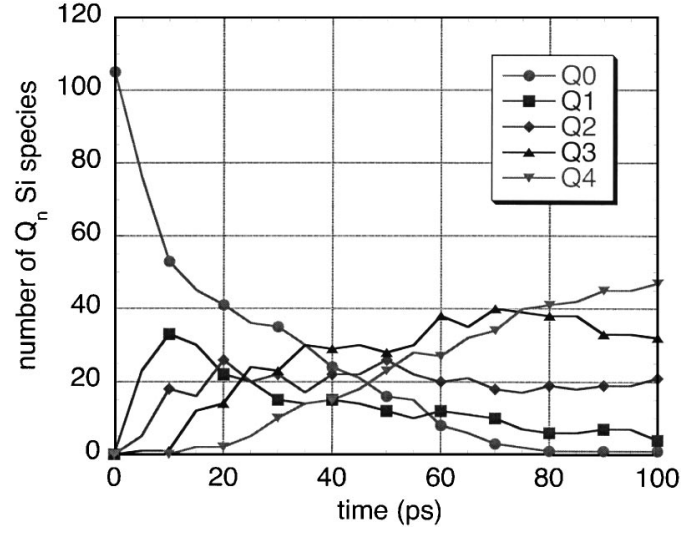

FIG. 3. Number of $Q_{n}$ silicon species vs simulation time (system density $=1.0 \mathrm{~g} / \mathrm{cm}^{3}$; the subscript $n$ indicates the number of bridging oxygen atoms attached to a given $\mathrm{Si}$ atom).

overall dimensions of our simulation ensembles $(<50 \AA)$ may affect the development of such "primary particles.",34

The time evolution of $Q_{n}$ silicon species (where the subscript $n$ indicates the number of bridging oxygen atoms attached to a given Si atom) exhibits interesting features. Figure 3 shows this evolution for the $1.0 \mathrm{~g} / \mathrm{cm}^{3}$ configuration. As might be expected, the number of $Q_{0}$ species decreases almost uniformly with time. The evolution of $Q_{1}$ species shows a peak at around $10 \mathrm{ps}$, followed in time by a peak in the number of $Q_{2}$ species. The number of $Q_{3}$ species peaks even later; while that of $Q_{4}$ species, expectedly, increases monotonously. This behavior is remarkably similar to results from the MD simulations of Garofalini and Martin, ${ }^{3}$ including the location of the $Q_{1}$ peak. These authors also point out the qualitative similarities of this behavior to data obtained from NMR investigations. ${ }^{35}$ It is worth noting here that a NMR study of the sol-gel polymerization of TEOS at $21{ }^{\circ} \mathrm{C}$ found the degree of condensation, defined in terms of the relative concentrations of $Q_{n} \mathrm{Si}$ species, to saturate at a value of $\sim 84 \% .^{36}$ This is a close match to the $83-87 \%$ completion observed in our simulations.

We have plotted pair correlation functions (PCFs) to examine the short-range order characteristics of the simulated structures at the end of the polymerization process. The $\mathrm{Si}-\mathrm{O}$ PCF [Fig. 4(a)] shows first-neighbor and second-neighbor peaks at $\sim 0.15$ and $\sim 0.4 \mathrm{~nm}$ respectively, while the $\mathrm{O}-\mathrm{O}$ PCF [Fig. 4(b)] has a first peak at $\sim 0.25 \mathrm{~nm}$ corresponding to the side length of a tetrahedral unit that characterizes the silica structure, followed by a second one at $\sim 0.5 \mathrm{~nm}$, which marks the distance between two far corners of corner-sharing tetrahedra. All of these values closely match x-ray diffraction measures for melt-derived silica glass and silica gels produced by the sol-gel method. ${ }^{37}$

Finally, we examine to what extent our simulated aerogels exhibit fractal geometry, and how this characteristic compares to a number of experimental studies. ${ }^{19-22}$ At gel densities comparable to our simulations, the range of selfsimilarity typically observed in experiments is about $10 \AA$ $<r<50 \AA$ (density $\left.=0.65 \mathrm{~g} / \mathrm{cm}^{3}\right){ }^{38}$ Our simulations produced percolating porous silica structures extending to about 36-42 $\AA$, with gel backbone densities ranging from 0.66 to $1.24 \mathrm{~g} / \mathrm{cm}^{3}$. (We exclude water molecules from den-
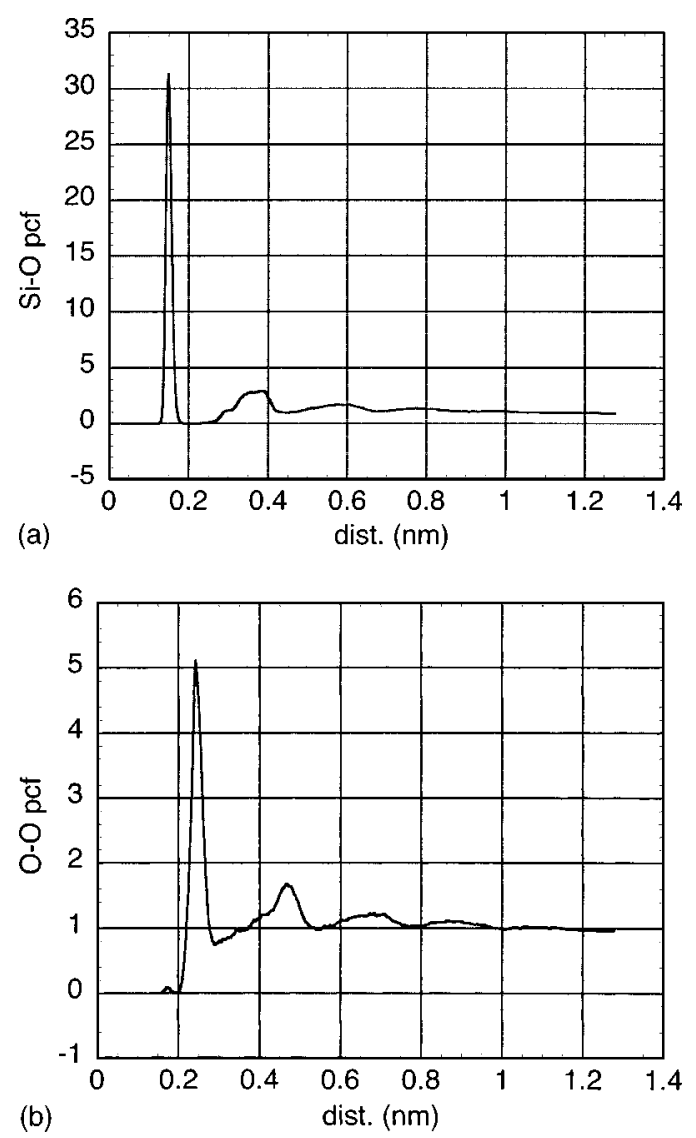

FIG. 4. (a) Si-O pair correlation function; (b) $\mathrm{O}-\mathrm{O}$ pair correlation function at end of polymerization process $\left(\right.$ system density $\left.=1.0 \mathrm{~g} / \mathrm{cm}^{3}\right)$.

sity calculations from this point onwards, so as to enable comparison with experiments on dried aerogels.) These structures are thus large enough for observation of fractal scaling behavior. To calculate the fractal dimension, we followed the methodology used by Kieffer and Angell, ${ }^{39}$ who derived the following expression for the fractal dimension $d_{f}$ of a simulated structure:

$$
d_{f}=\frac{d \ln N(r)}{d \ln r} .
$$

Here, $N(r)$ is the number of ions surrounding a central ion at distance $r$-essentially the space integral of the pair correlation function. Figure 5 shows representative $\log -\log$ plots of $N_{\mathrm{Si}-\mathrm{Si}}(r)$ vs $r$ at various densities [only the smooth portions of the curves beyond the near-neighbor peaks are shown: this is where Eq. (2) applies]. The fractal dimension $d_{f}$ is evaluated as the slope of these plots (we have considered Si ions only in this calculation) and is shown plotted against density in the inset to Fig. 5. All values of $d_{f}$ lie between 2.0 and 3.0 - thereby confirming that the structures are indeed fractal.

Having established the fractal nature of our simulated gels, we now want to determine whether highly porous silica at a given density has a unique structure or whether it depends on the assembly process. To this end we compare the structural features of configurations prepared by simulated gelation reactions described above with those of porous silica produced by isotropically rupturing a dense glass. Spe- 


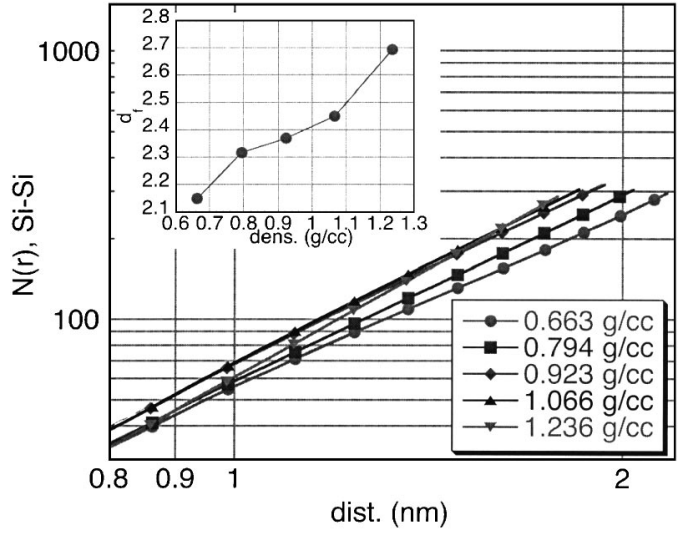

FIG. 5. Calculation of fractal dimension from slope of $N_{\mathrm{Si}-\mathrm{Si}}(r)$ vs $r$; inset, fractal dimension vs density.

cifically, we are interested in how the intermediate-range order characteristics, as reflected in the fractal dimension evaluated from $N_{\mathrm{Si}-\mathrm{Si}}(r)$, might differ in the two cases. Furthermore, by monitoring the fractal dimension, we can assess the structural changes that occur during the density springback as a result of drying the hydrogels (polymerized gel before water extraction) or releasing the tensile stress, respectively.

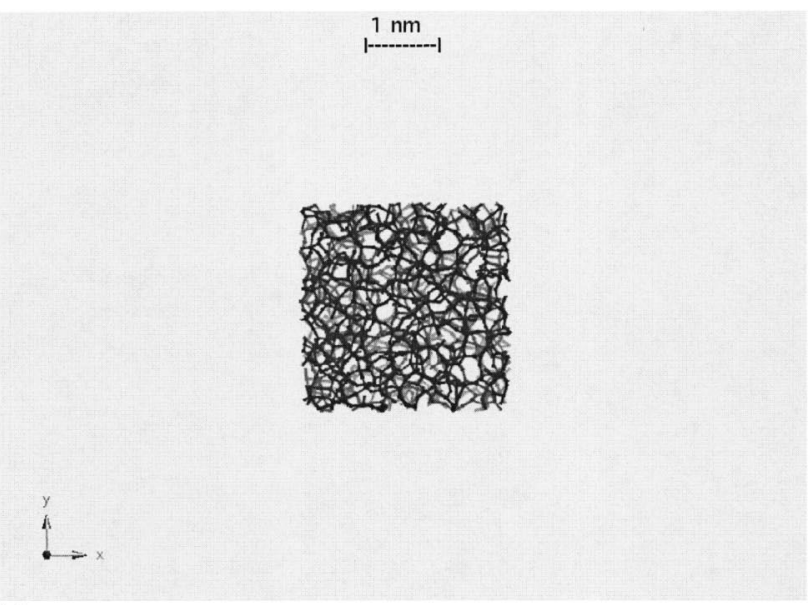

(a)

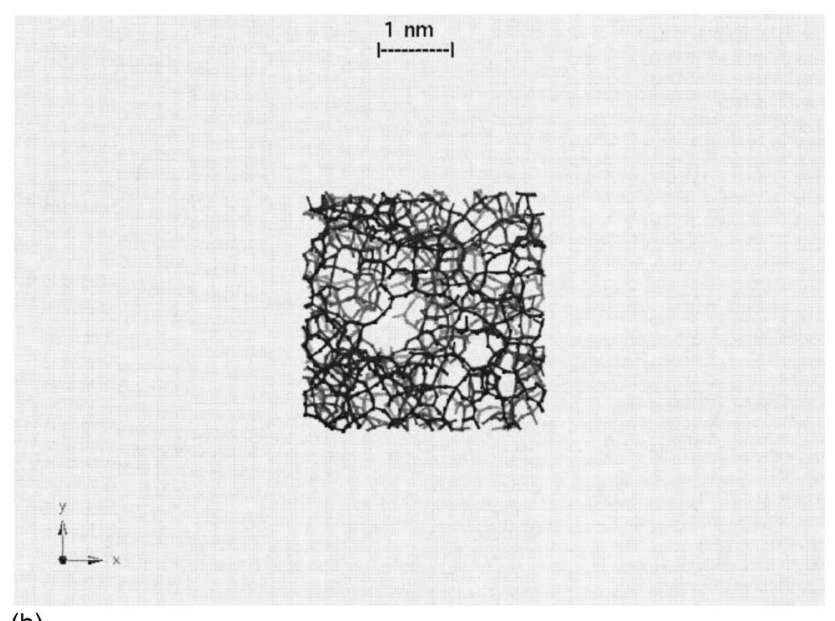

(b)

FIG. 6. Two-dimensional (2D) projections of structures corresponding to three different steps in expansion of silica glass from 2.2 to $0.2 \mathrm{~g} / \mathrm{cm}^{3}$ (ruptured system): (a) $2.2 \mathrm{~g} / \mathrm{cm}^{3}$ (before expansion); (b) $1.4 \mathrm{~g} / \mathrm{cm}^{3}$; (c) $0.8 \mathrm{~g} / \mathrm{cm}^{3}$; (d) $0.2 \mathrm{~g} / \mathrm{cm}^{3}$. To better reveal the network structure these images only show the $\mathrm{Si}-\mathrm{O}$ bonds. $\mathrm{Si}$ atoms are located at the vertices of four converging bonds and $\mathrm{O}$ atoms at the kinks in between two vertices.
To create ruptured silica structures, a configuration of 512 silicon atoms (as in the condensation reaction simulations) and 1024 oxygen atoms was melted at an elevated temperature of $6000 \mathrm{~K}$, and then quenched to room temperature yielding a glass with a density of $2.2 \mathrm{~g} / \mathrm{cm}^{3}$. (There are no hydrogen atoms in this model.) The simulation box was then uniformly expanded to reduce the density in steps of $0.2 \mathrm{~g} / \mathrm{cm}^{3}$ till we obtained a system density of $0.2 \mathrm{~g} / \mathrm{cm}^{3}$. The start and end configurations, along with a couple of intermediate steps, are illustrated in Figs. 6(a)-6(d). As before, the fractal dimension $d_{f}$ is calculated from the $N_{\mathrm{Si}-\mathrm{Si}}(r)$ vs $r$ curves.

The solid lines in Fig. 7 show $d_{f}$ plotted against density for both the reaction-generated and ruptured structures before they are relaxed. For the porous structures formed by rupture, $d_{f}$ is seen to remain leveled at 3.0 until the tensile limit of the glass is reached at about $1.6 \mathrm{~g} / \mathrm{cm}^{3}$ and then drop smoothly from 3.0 to about 2.0 - the structures becoming more tenuous (smaller $d_{f}$ ) as density is lowered to $0.2 \mathrm{~g} / \mathrm{cm}^{3}$. The curve is comparable to results from previous simulations, even though these earlier studies were carried out using a different potential. ${ }^{39}$ The reaction-generated configurations, which cover a smaller density range, have fractal dimensions that are consistently lower than those of the rup-

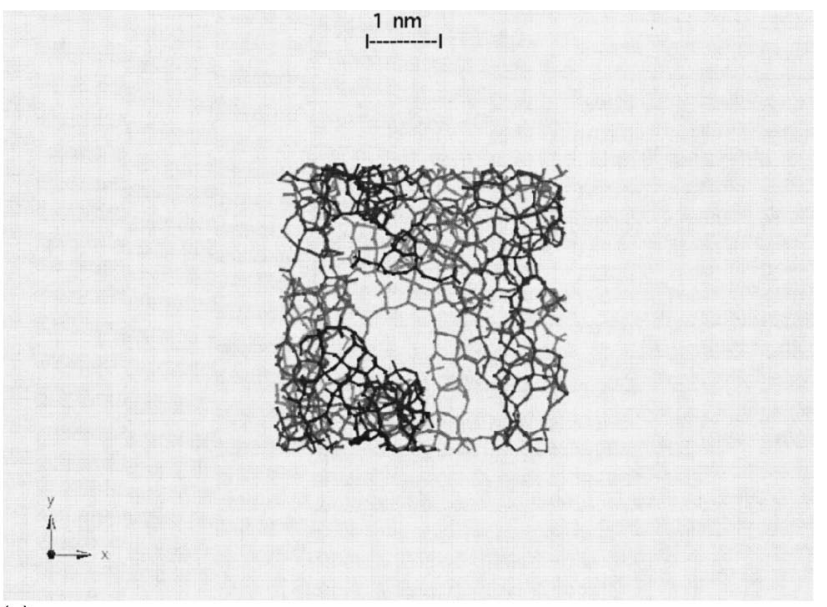

(c)

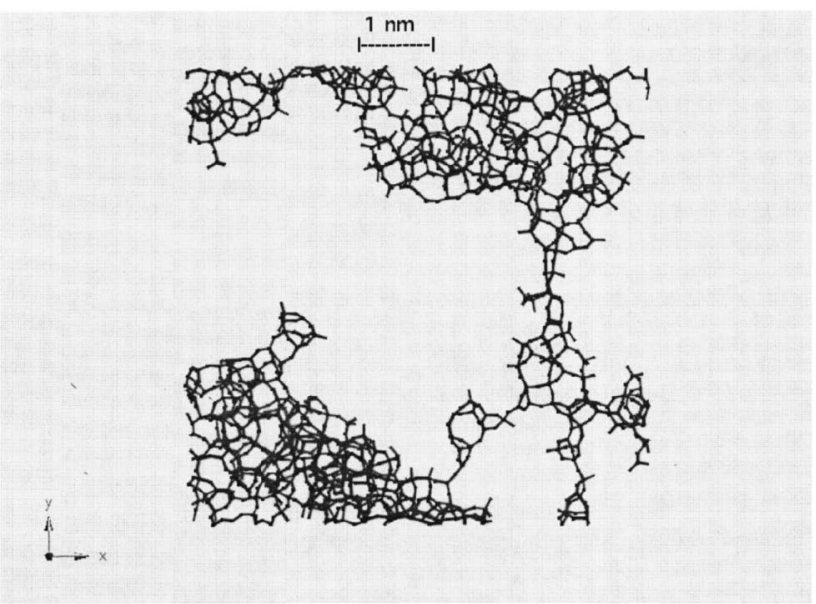

(d) 


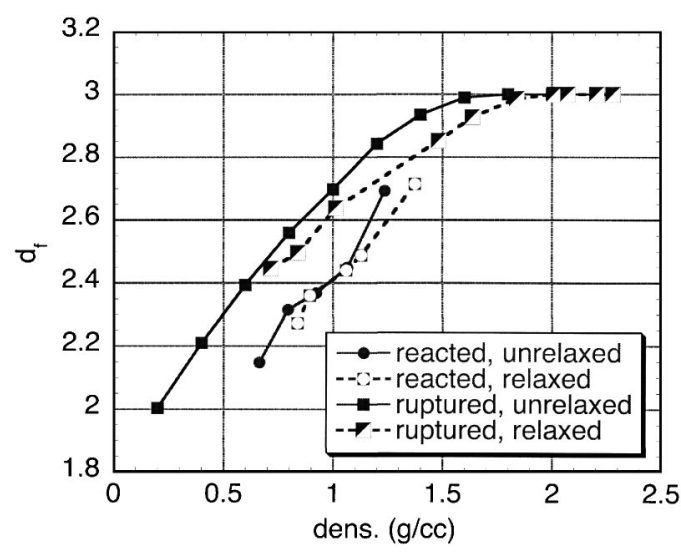

FIG. 7. Effect of relaxation on fractal dimension of porous silica for reacted and ruptured systems.

tured glass at comparable densities. This indicates fundamental structural differences between the silica gel produced by sol-gel condensation and porous structures that may result from mechanically ruptured glass. Apparently, there are some basic differences in the way porosity is generated in the two cases: in the reacted system, the pores result from the need to accommodate the water molecules that form during condensation, whereas in the ruptured system pores open up because of the inability of the system to maintain cohesion in all dimensions. In one case the system deals with an excess of a secondary phase (water), in the other with a lack of primary phase (polymerized gel). Hence, the governing force balance in the two situations is different. While for both systems the silicon-oxygen bonds at the internal pore surfaces are under tension upon formation of the fractal network (this will become obvious in the following discussion), in case of the reaction-generated structures this tension is induced by the pressure from the condensation of water within the pores, whereas in the case of the ruptured structures a tensile stress is externally applied to the system.

Interestingly, while in both cases, $d_{f}$ lies between 2.0 and 3.0, which confirms fractal scaling behavior, the two-phased system produces a gel structure with a lower value of $d_{f}$ for any given density than the ruptured system. Recall that fractality implies self-similarity of structural features within the range over which fractal scaling applies. This means that in this regime pores of all sizes exist and that they have similar geometry irrespective of their size. Hence, the fact that the fractal dimension of the reaction-generated structure is lower than that of the ruptured structure reveals another interesting detail about the process by which these structures develop. Fracture in the latter case is initiated at a particular site in the structure, i.e., the weakest link. A fissure is nucleated at this site and continues to grow as the configuration is strained. Other voids nucleate subsequently and in terms of size these evolve with delay, never catching up to the initial crevice. This explains the distribution in void sizes. Furthermore, subsequent fissures tend to emanate from the extremities of existing voids, as it occurs during the bifurcation of cracks. The overall progression of void formation can best be described as a nucleation and growth process, and the majority of the pore volume can be attributed to the void at which the failure of the structure initiated. ${ }^{39}$
Conversely, condensation reactions occur sporadically. The locations of consecutive reactions are spatially uncorrelated, as would be expected of a reaction-limited aggregation. With time, pockets form to hold the condensed water, and they have sizes that are independent of each other. Apparently, there is no preferred size for such pores and they have similar shape. This results in an even pore size distribution and may explain the lower fractal dimension. In spite of the differences in the actual values of $d_{f}$, it is noteworthy that the slopes of the fractal dimension vs density plots in Fig. 7 are comparable for the reaction-generated and ruptured systems. Seemingly, the magnitude of the fractal dimension of a gel backbone depends on the process by which it is formed. However, the relative change in $d_{f}$ that is associated with the redistribution of matter upon changing the density is not affected by the synthesis process, possibly because it is a measure that depends on the way structural building blocks can connect to each other to span space.

Aerogels are prepared in the laboratory by removing the pore fluid from hydrogels under supercritical conditions. Such conditions are necessary to eliminate the formation of a meniscus in the fluid at the pore mouths, because the tensile stress exerted by the meniscus invariably causes the gel backbone to collapse. Even when carrying out the fluid removal under supercritical conditions, a small amount of shrinkage is often observed during this procedure, and it is not entirely clear how this shrinkage might affect the fractal dimension of the gel structure. In order to compare the characteristics of our simulated gels to those of experimental aerogels, and in order to assess the structural changes that may occur during supercritical drying, we need to remove the condensed water molecules and allow the structures to relax to a stable state under normal atmospheric conditions (i.e., approximately zero pressure).

We followed two approaches to simulate the interfacefree fluid removal: one is instantaneous and the other one gradual. In the first approach, we removed the water molecules from the reacted system in a one-step extraction process. Then the system was relaxed to zero pressure with a $10 \mathrm{ps}$ constant-pressure MD run, followed by a 5 ps constant-volume run and a second constant-pressure run for $10 \mathrm{ps}$, to ensure a stable structure. The ruptured glass was subjected to a similar three-step relaxation procedure for comparison purposes. The results are shown by the dotted lines in Fig. 7. As we can see, there is a considerable springback associated with the ruptured glasses-they are relatively unstable as formed (under large negative pressures), and coil back to more dense configurations with higher fractal dimension as the tensile stress is released. The lower the initial density, the larger is the extent of spring-back. By comparison, the reaction-generated gel structures are more stable and robust to relaxation-the spring-back is much lower in this case.

However, the one-step extraction of water from the reacted system described above may be a rather crude approximation of supercritical drying. A better model of the process would be a gradual removal of water from the simulation box, accompanied by a simultaneous relaxation to zero pressure. The passage of a curvature-free liquid-vapor interface 


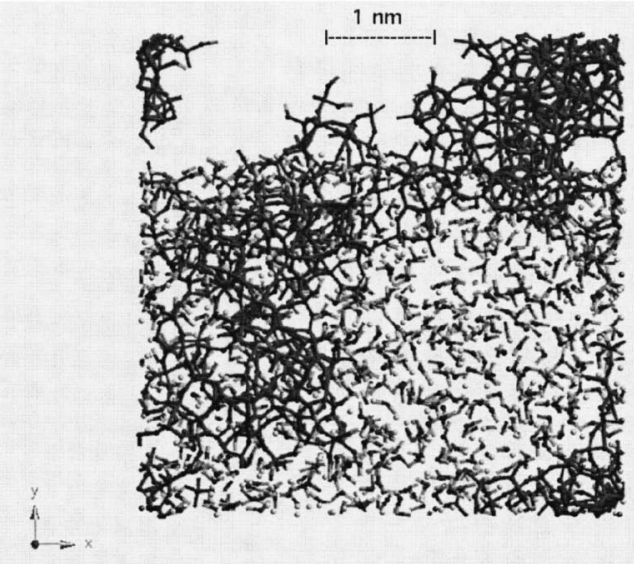

(a)

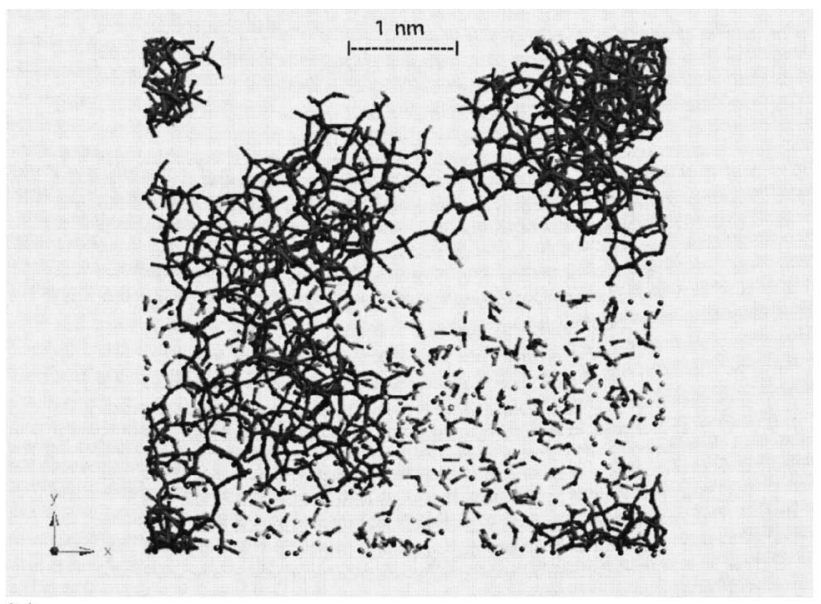

(b)

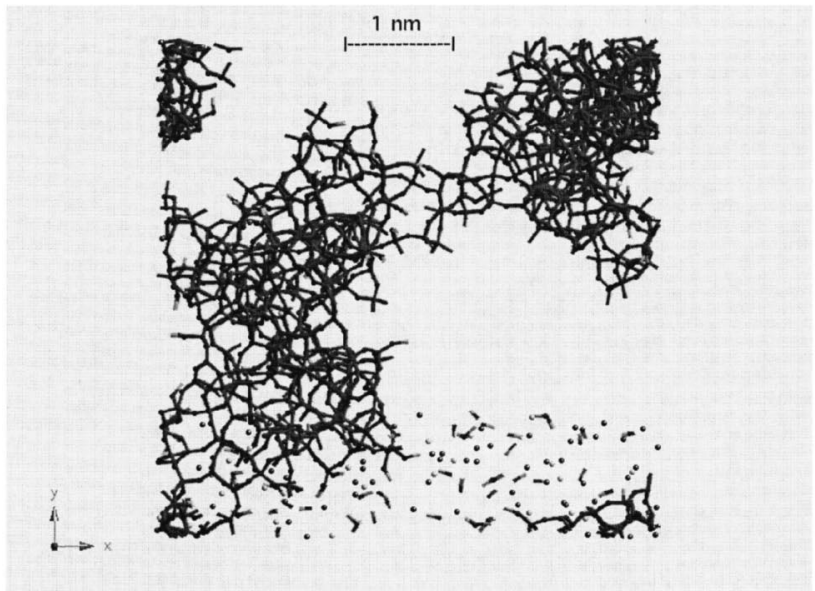

(c)

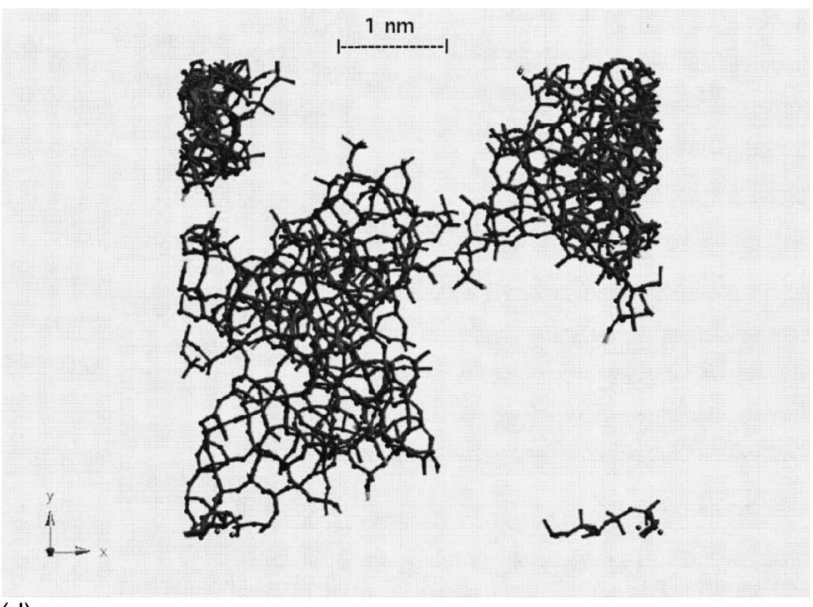

(d)

FIG. 8. (a)-(d) 2D projections of structures corresponding to four different stages in gradual extraction of water molecules from reacted gel. The silica network bonds are shown as dark lines, as in Fig. 6. Water molecules can be identified as a kinked pair of bonds with two light-colored beads at the extremities and hydroxyl groups as one half of these structures.

through the pores of our simulated structure was implemented by successive removal of condensed water molecules from the top one-fourth of the simulation box, then from the top half, and so on, continuing progressively downwards until the system was totally free of water [see Figs. 8(a)-8(d)]. Each stage of water removal was followed by a three-step relaxation to zero pressure, as previously described. During this relaxation, thermal motion of water molecules rapidly created a diffused liquid-vapor interface. Figure 9 compares the effects of the two different "drying" procedures - the corresponding configurations are numbered for identification. As can be seen from the plots, the gradual drying process has a much smaller effect both on the density and the fractal dimension than the one-step water extraction.

Plots of scattering intensity obtained from SANS experiments on neutrally reacted and dried silica aerogels yield a fractal dimension $d_{f}$ of $\sim 2.4$ over the density range $0.11-0.36 \mathrm{~g} / \mathrm{cm}^{3}{ }^{40}$ An identical measure has been reported from SAXS studies. ${ }^{41}$ We observe approximately the same fractal dimensions for simulated structures of somewhat higher densities; i.e., $d_{f}$ subsequent to gradual drying varies from 2.2 to 2.63 (Fig. 9), corresponding to gel densities of $0.7-1.2 \mathrm{~g} / \mathrm{cm}^{3}$. As has been pointed out in the literature
(Ref. 2, p. 535), it is difficult to ascertain whether the fractal dimension measured on a dried aerogel truly corresponds to that of the original hydrogel structure as formed at the gelation point. Our simulations indicate that this difference is not appreciable, i.e., the shrinkage involved in the drying process does not have a significant effect on the value of $d_{f}$.

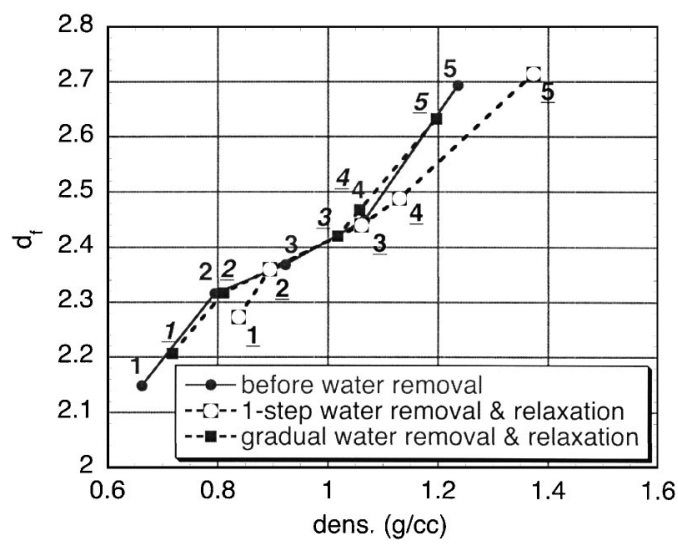

FIG. 9. Effect of drying procedure on fractal dimension of reacted gel. 


\section{CONCLUSIONS}

We have successfully simulated the sol-gel condensation process with a reactive three-body charge-transfer MD potential to obtain polymerized silica aerogels. From various measures, the structures are seen to be realistic as compared with experiments. Comparison of their fractal dimension $d_{f}$ with that of a porous silica structure generated by rupture of dense silica reveals that $d_{f}$ is not uniquely related to the gel backbone density, and consequently the degree of porosity of a structure, but that this dimension depends on the process by which the structure is created. The supercritical drying process is modeled both by a one-step and a gradual removal of water molecules followed by the relaxation of the aerogels to zero pressure. The final fractal dimension of the gels is seen to vary between 2.2 and 2.63-a close match with the experimentally observed value of 2.4 for neutrally reacted gels. Our simulations show that the drying process has a negligible effect on the fractal nature of the silica structures formed at the gelation point.

\section{ACKNOWLEDGMENTS}

This work was supported by grants from the Petroleum Research Fund of the American Chemical Society, and the National Science Foundation. The authors would like to thank Liping Huang and Feng Qi of the University of Michigan, Ann Arbor, for assistance with the project.

${ }^{1}$ J. Zarzycki, J. Sol-Gel Sci. Technol. 8, 17 (1997).

${ }^{2}$ C. J. Brinker and G. W. Scherer, Sol-Gel Science: The Physics and Chemistry of Sol-Gel Processing (Academic, New York, 1990).

${ }^{3}$ S. H. Garofalini and G. Martin, J. Phys. Chem. 98, 1311 (1994).

${ }^{4}$ B. P. Feuston and S. H. Garofalini, J. Phys. Chem. 94, 5351 (1990).

${ }^{5}$ B. P. Feuston and S. H. Garofalini, Chem. Phys. Lett. 170, 264 (1990).

${ }^{6}$ G. E. Martin and S. H. Garofalini, J. Non-Cryst. Solids 171, 68 (1994).

${ }^{7}$ J. C. G. Pereira, C. R. A. Catlow, and G. D. Price, J. Phys. Chem. A 106, 130 (2002).

${ }^{8}$ N. A. Peppas, A. B. Scranton, A. H. Tsou, and D. E. Edwards, in Better Ceramics Through Chemistry III, edited by C. J. Brinker, D. E. Clark, and D. R. Ulrich (Material Research Society, Pittsburgh, PA, 1988), Vol. 121, p. 43.

${ }^{9}$ R. A. Assink and B. D. Kay, J. Non-Cryst. Solids 99, 359 (1988).
${ }^{10}$ N. Re, J. Non-Cryst. Solids 142, 1 (1992).

${ }^{11}$ B. D. Kay and R. A. Assink, J. Non-Cryst. Solids 104, 112 (1988).

${ }^{12}$ A. Hasmy and R. Jullien, J. Non-Cryst. Solids 186, 342 (1995).

${ }^{13}$ P. I. Pohl, J. L. Faulon, and D. M. Smith, J. Non-Cryst. Solids 186, 349 (1995).

${ }^{14}$ E. A. Chandler and D. F. Calef, J. Non-Cryst. Solids 186, 356 (1995).

${ }^{15}$ L. Duffrene and J. Kieffer, J. Phys. Chem. Solids 59, 1025 (1998).

${ }^{16}$ L. P. Huang and J. Kieffer, J. Chem. Phys. 118, 1487 (2003).

${ }^{17}$ L. P. Huang and J. Kieffer, Phys. Rev. B 69, 224203 (2004).

${ }^{18}$ L. P. Huang and J. Kieffer, Phys. Rev. B 69, 224204 (2004).

${ }^{19}$ D. W. Schaefer and K. D. Keefer, Phys. Rev. Lett. 56, 2199 (1986).

${ }^{20}$ R. Vacher, T. Woignier, J. Pelous, and E. Courtens, Phys. Rev. B 37, 6500 (1988).

${ }^{21}$ F. Devreux, J. P. Boilot, F. Chaput, and B. Sapoval, Phys. Rev. Lett. 65, 614 (1990)

${ }^{22}$ E. Courtens and R. Vacher, in Fractals in the Natural Sciences, edited by M. Fleischmann, D. J. Tildesley, and R. C. Ball (Princeton University Press, Princeton, NJ, 1989), p. 55

${ }^{23}$ A. Hasmy, M. Foret, J. Pelous, and R. Jullien, Phys. Rev. B 48, 9345 (1993).

${ }^{24}$ A. Hasmy, E. Anglaret, M. Foret, J. Pelous, and R. Jullien, Phys. Rev. B 50, 6006 (1994)

${ }^{25}$ M. Grzegorczyk, M. Rybaczuk, and K. Maruszewski, Chaos, Solitons Fractals 19, 1003 (2004).

${ }^{26}$ A. C. Lasaga and G. V. Gibbs, Phys. Chem. Miner. 14, 107 (1987).

${ }^{27}$ M. J. Sander, M. Leslie, and C. R. A. Catlow, J. Chem. Soc., Chem. Commun. 19, 1271 (1984).

${ }^{28}$ C. R. A. Catlow and A. N. Cormack, Int. Rev. Phys. Chem. 6, 227 (1987).

${ }^{29}$ M. L. Huggins and J. E. Mayer, J. Chem. Phys. 1, 643 (1933).

${ }^{30}$ P. P. Ewald, Ann. Phys. (Leipzig) 64, 253 (1921).

${ }^{31}$ C. Kittel, Introduction to Solid State Physics (Wiley, New York, 1996).

${ }^{32}$ R. K. Iler, The Chemistry of Silica (Wiley, New York, 1979).

${ }^{33}$ L. L. Hench and J. K. West, Chem. Rev. (Washington, D.C.) 90, 33 (1990).

${ }^{34}$ G. Beaucage, Phys. Rev. E 70, 031401 (2004).

${ }^{35}$ F. Devreux, J. P. Boilot, F. Chaput, and A. Lecomte, Phys. Rev. A 41, $6901(1990)$.

${ }^{36}$ L. Malier, J. P. Boilot, F. Chaput, and F. Devreux, Phys. Rev. A 46, 959 (1992).

${ }^{37}$ K. Kamiya, T. Dohkai, M. Wada, T. Hashimoto, J. Matsuoka, and H. Nasu, J. Non-Cryst. Solids 240, 202 (1998).

${ }^{38}$ I. Beurroies, L. Duffours, P. Delord, T. Woignier, and J. Phalippou, J. Non-Cryst. Solids 241, 38 (1998)

${ }^{39}$ J. Kieffer and C. A. Angell, J. Non-Cryst. Solids 106, 336 (1988).

${ }^{40}$ T. Woignier, J. Phalippou, and R. Vacher, in Better Ceramics Through Chemistry III, edited by C. J. Brinker, D. E. Clark, and D. R. Ulrich (Material Research Society, Pittsburgh, PA, 1988), Vol. 121, p. 697.

${ }^{41}$ T. Woignier, J. Reynes, A. H. Alaoui, I. Beurroies, and J. Phalippou, J. Non-Cryst. Solids 241, 45 (1998). 